\title{
Acute Hemorrhagic Encephalomyelitis Following Pneumococcal Meningitis in a 19 Years Old Girl
}

\section{Asadollahzade E${ }^{1 *}$, Johari $\mathrm{MS}^{2}$ and Hesami $0^{1}$}

${ }^{1}$ Department of Neurology, Imam Hossein Hospital, Shahid Beheshti University of Medical Sciences, Iran

${ }^{2}$ Department of Radiology, Iran University of Medical Sciences, Iran

\section{Case Report}

Volume 2 Issue 2

Received Date: June 23, 2018

Published Date: July 13, 2018

*Corresponding author: Elnaz Asadollahzade, Department of Neurology, Imam Hossein Hospital, Shahid Beheshti University of Medical Sciences, Tehran, Iran, Tel: 09126444577; Email: drelnazasadollahzade@gmail.com

\section{Abstract}

Acute hemorrhagic encephalomyelitis (AHEM) is a rare fulminant subtype of acute disseminated encephalomyelitis (ADEM). ADEM is characterized by multifocal neurological deficit, loss of consciousness and fever. These are commonly occurred following viral or bacterial infections or vaccination in children. The focal intensities representing of inflammation and hemorrhages in MRI are so valuable for diagnosis of AHEM. Here, we present a case of AHEM following alpha-hemolytic streptococci meningitis presented with fever and loss of consciousness. She was first treated with antibiotics but owing to recurrence of loss of consciousness, she undergone serial MRIs. T1W high signal intensities in her white matter were suggestive of AHEM. The patient was treated with high-dose of methylprednisolone and IVIg and then she had gotten awake and her symptoms had revealed.

Keywords: Acute Hemorrhagic Encephalomyelitis; Acute Disseminated encephalomyelitis; Alpha-Hemolytic Streptococci; Meningitis; Loss Of Consciousness

\section{Abbreviations: AHEM: Acute Hemorrhagic Encephalomyelitis; ADEM: Acute Disseminated Encephalomyelitis; MRI: Magnetic Resonance Imaging; AHLE: Acute Hemorrhagic Leukoencephalitis; CNS: Central Nervous System; CMV: Cytomegalovirus.}

\section{Introduction}

Weston Hurst was the first clinician who described acute hemorrhagic encephalomyelitis (AHEM) in 1941. It also known as acute hemorrhagic leukoencephalitis (AHLE), Hurst disease or Weston-Hurst syndrome .This central nervous system (CNS) demyelinating immunemediated inflammatory disease is the rare and fulminant variant of acute disseminated encephalomyelitis (ADEM).ADEM is usually transient and self-limiting, and diagnosed by acute or subacute encephalopathy [1-3].

ADEM is usually diagnosed in children from 5 to 8 years, but it can be seen in any age [4]. It is often 


\section{Clinical Radiology \& Imaging Journal}

happened after viral infections and vaccination. The viral and bacterial infections which are supposed to be related with ADEM are including influenza virus, enterovirus, measles, mumps, rubella, varicella-zoster, Epstein-Barr virus, cytomegalovirus (CMV), herpes simplex virus, hepatitis A, coxsackievirus, Mycoplasma pneumoniae, Borrelia burgdorferi, Leptospira and beta-hemolytic Streptococcus. Post vaccination ADEM has been related with hepatitis B, pertussis, diphtheria, measles, mumps, rubella, pneumococcus, varicella, influenza, Japanese B encephalitis, smallpox, poliomyelitis, and human papillomavirus. However, discovery of recombinant vaccines instead of vaccines from CNS cultures has reduced the risk of ADEM.

Furthermore, the occurrence of AHEM has been reported following infection of upper respiratory tract and influenza [5,6].

The first clinical signs of ADEM are commonly initiate 2 to 14 days after infection or vaccination. These include fever, malaise, headache, nausea, vomiting and rapid progressing encephalopathy which leads to loss of consciousness and death in few days $[7,8]$.

Magnetic resonance imaging (MRI) is so valuable for characterization of ADEM and AHLE. The MRI of patients with ADEM revealed predominant demyelination of white matter in brain and spinal cord $[3,7,9]$.

In this article, we present a case of AHEM following alpha-hemolytic streptococci meningitis.

\section{Case Report}

The patient was a 19 years old woman with presentation of fever and loss of consciousness. She has fever, nausea, vomiting and earache since 2 weeks before visit. Moreover, she had one episode of generalized tonic clonic seizure lasted around 1 minute a day before visit and after that she had stupor and had not properly respond to stimulants. She had past medical history of neuro developmental delay and had studied in exceptional school. In addition, she had two episodes of generalized tonic clonic seizure when she was 9 years old and was treated with sodium valproate $200 \mathrm{mg}$ twice per day.

In primary visit she had impaired consciousness presented with drowsiness and loss of obeying from orders. In physical examination, she had neck stiffness. Her axillary temperature was $40^{\circ} \mathrm{C}$, blood pressure of $100 / 50$ and pulse rate $90 / \mathrm{min}$.
Owing to loss of consciousness, a brain CT-scan was first achieved and as there was no contraindication, she underwent lumbar puncture which the results were as followed: white blood cell $162 / \mu \mathrm{l}$, protein $122 \mathrm{mg} / \mathrm{dl}$, glucose $11 \mathrm{mg} / \mathrm{dl}$ and red blood cell $124 / \mu \mathrm{l}$. CSF culture was positive for alpha-hemolytic streptococcus (Gram positive cocci). Therefore, with diagnosis of streptococcal bacterial (pneumococci) meningitis, she was treated with Ceftriaxone 2 gr twice daily, Vancomycin 1 gr twice daily and dexamethasone 4 gr three times daily. In addition, she was treated with one loading dose of Phenytoin $1 \mathrm{gr}$ IV) and then maintenance of phenytoin (100 g IV three times per day). Nevertheless, she had progressing loss of consciousness and so intubated and admitted in ICU. Her electroencephalogram showed "generalized slow wave background without epileptiform discharge". Therefore, due to loss of improvement in her consciousness despite of antibiotic and anti-epileptic treatments, she underwent brain MRI with contrast. The results of first MRI (Figure 1) showed evidences of abnormal signals in bilateral periventricular and subcortical white matter and also basal ganglia, brain stem and corpus callosum without enhancement in favor of acute disseminated encephalomyelitis (ADEM). Hence, the patient was treated with pulse of methylprednisolone $(1 \mathrm{gr}$ daily for 5 days) in addition to previous treatments. Her consciousness was improved, awaked and had the ability of obeying the orders. So, she was extubated and transferred to ward.

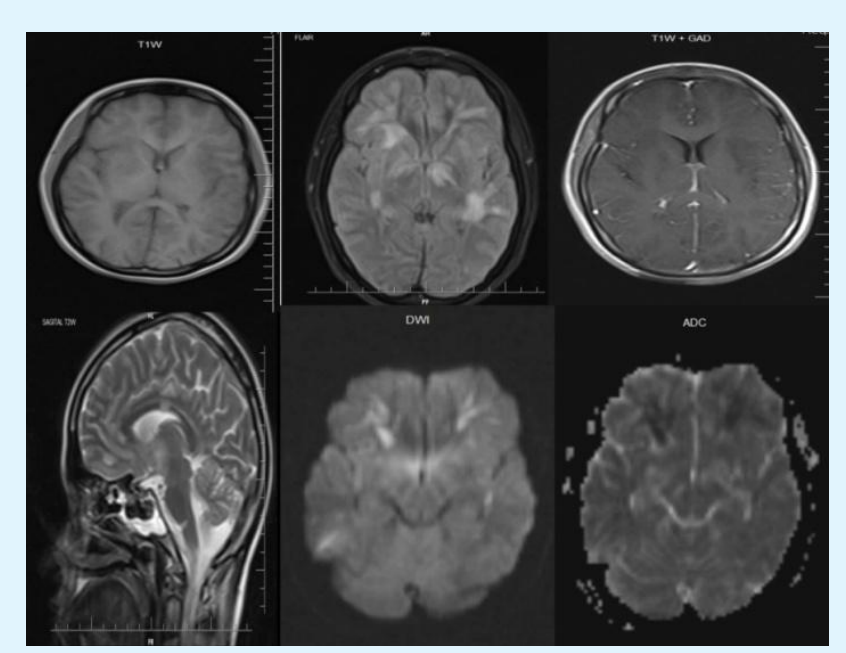

Figure 1 (First MRI): Multiple high signal intensities are seen in bilateral periventricular, subcortical white matter, basal ganglia, brain stem and carpus callosum without enhancement with some of which show restriction on DWI. 


\section{Clinical Radiology \& Imaging Journal}

After admitting in the ward, she had recurrent loss of consciousness. Thus, the patient undergone secondary brain MRI (Figure 2) which showed enhanced lesions and a hemorrhagic focus in the frontal subcortical white matter. The patient was gotten under treatment with prednisolone maintenance besides previous treatments and her consciousness was improved. However, she had loss of consciousness again and intubated.

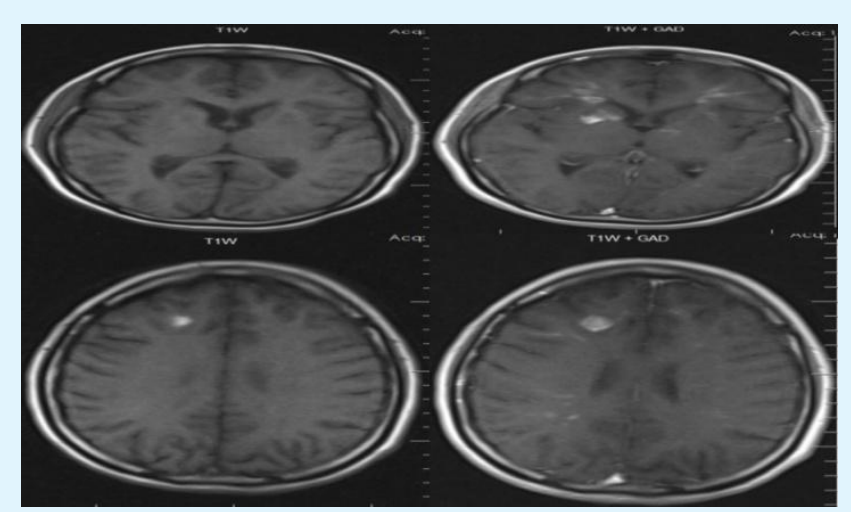

Figure 2 (Second MRI): In comparison with previous study, plaques show enhancement and a focus of T1W hyper intensity in right frontal subcortical white matter suggesting intra-parenchymal hemorrhage.

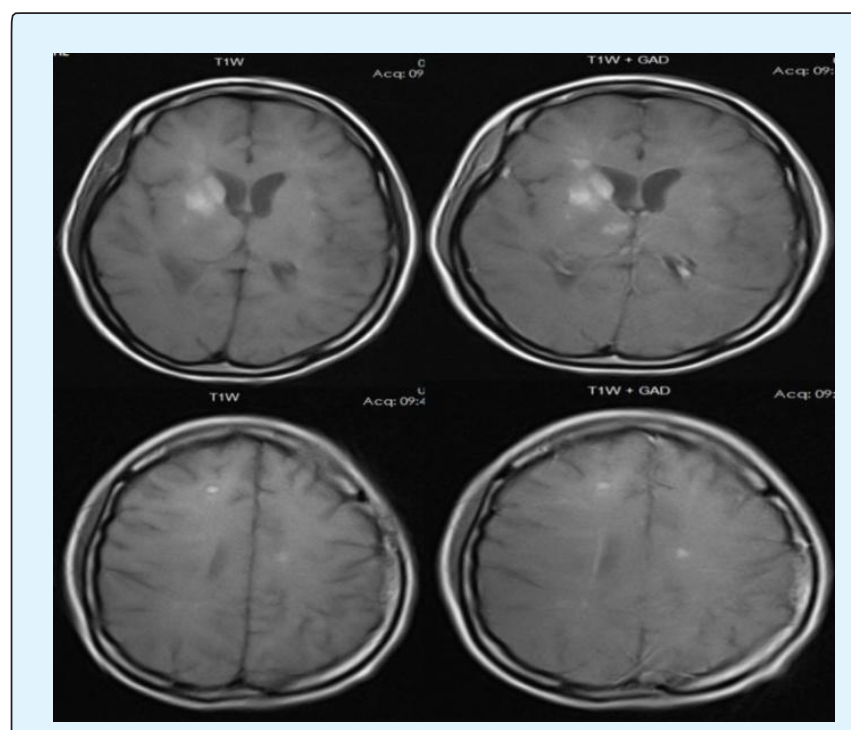

Figure 3 (Third MRI): More plaques in periventricular and subcortical white matter and basal ganglia show T1W hyper intensity in favor of hemorrhagic transformation but in comparison with previous MRI, the number of plaques and the enhancement are regressed. Also, the size of mentioned intraparenchymal hemorrhages in right frontal lobe was decreased.
The third MRI (Figure 3) revealed that most of lesions developed to be hemorrhagic. Thus, with probable diagnosis of acute hemorrhagic leukoencephalitis (AHEM), the patient treated with IVIG, 20 gr daily for 5 days. Her consciousness was improved slowly and extubated. And then her eye and verbal contact were enhanced and so discharged.

\section{Discussion}

Here we present a case of 19 years old girl presented with fever, loss of consciousness and neck stiffness which was primarily diagnosed as alpha-hemolytic streptococci meningitis. Following antibiotic treatments, the patient undergone severe loss of consciousness and intubated. Serial MRIs showed enhanced lesions which developed to be hemorrhagic in few days and the patients were diagnosed with acute hemorrhagic encephalomyelitis and treated with high dose of prednisolone and IVIG.

There is no consensus for treatment of AHEM and the gathered information from experts and case series are the only references. Invasive management of acute attacks of ADEM including critical care is vital. It is recommended to early administrating acyclovir $(30 \mathrm{mg} / \mathrm{kg} /$ day $)$ as antiviral therapy. Because viral infections such as herpes simplex are common primary diagnosis of children with fever and encephalitis. High dose corticosteroid therapy is the most prevalent treatment which has been reported in the literature. Short-term (3-5 days) high dose (20-30 $\mathrm{mg} / \mathrm{kg} /$ day to $1 \mathrm{~g} /$ day) I.V. infusion of methylprednisolone is mostly used. IVIg is the other choice which is administrated with or without corticosteroid. The total dose of IVIg is recommended to be $2 \mathrm{~g} / \mathrm{kg}$ infused over $2-5$ days $[4,7,9-11]$.

To the best of our knowledge, our case is the first reported case of AHEM following alpha-hemolytic streptococci meningitis. Close observation of patient and serial MRIs has led to development of diagnosis from usual meningitis to ADEM and then fulminant type of ADEM (AHEM).

\section{References}

1. Payne ET, Rutka JT, Ho TK, Halliday WC, Banwell BL (2007) Treatment leading to dramatic recovery in acute hemorrhagic leukoencephalitis. J Child Neurol 22(1):109-113.

2. Rahmlow MR, Kantarci O (2013) Fulminant demyelinating diseases. Neurohospitalist 3(2): 81-91. 


\section{Clinical Radiology \& Imaging Journal}

3. Wingerchuk DM (2003) Postinfectious encephalomyelitis. Curr Neurol Neurosci Rep 3(3): 256-264.

4. Dale RC, de Sousa C, Chong WK, Cox TC, Harding B, et al. (2000) Acute disseminated encephalomyelitis, multiphasic disseminated encephalomyelitis and multiple sclerosis in children. Brain 12: 2407-2422.

5. Noorbakhsh F, Johnson RT, Emery D, Power C (2008) Acute disseminated encephalomyelitis: clinical and pathogenesis features. Neurol Clin 26(3): 759-780.

6. Tenembaum SN (2013) Acute disseminated encephalomyelitis. Handb Clin Neurol 112: 12531262.

7. Tenembaum S, Chamoles N, Fejerman N (2002) Acute disseminated encephalomyelitis: a long-term followup study of 84 pediatric patients. Neurology 59(8): 1224-1231.
8. Leake JA, Albani S, Kao AS, Senac MO, Billman GF, et al. (2004) Acute disseminated encephalomyelitis in childhood: epidemiologic, clinical and laboratory features. Pediatr Infect Dis J 23(8): 756-764.

9. Hynson JL, Kornberg AJ, Coleman LT, Shield L, Harvey AS, et al. (2001) Clinical and neuroradiologic features of acute disseminated encephalomyelitis in children. Neurology 56(10): 1308-1312.

10. Tenembaum S, Chitnis T, Ness J, Hahn JS (2007) International Pediatric MSSG. Acute disseminated encephalomyelitis. Neurology 68(16): 23-36.

11. Pohl D, Tenembaum S (2012) Treatment of acute disseminated encephalomyelitis. Curr Treat Options Neurol 14(3): 264-275. 